\title{
Impact of the new FIGO 2013 classification on prognosis of stage I epithelial ovarian cancers
}

This article was published in the following Dove Press journal:

Cancer Management and Research

\author{
Céline Montavon Sartorius' \\ Uzma Mirza' \\ Andreas Schötzau \\ Gillian Mackay' \\ Daniel Fink ${ }^{3}$ \\ Neville F Hacker ${ }^{4}$ \\ Viola \\ Heinzelmann-Schwarz ${ }^{1,2}$ \\ 'Department of Gynecology and \\ Gynecological Oncology, Hospital for \\ Women, University Hospital Basel, \\ Basel, Switzerland; ${ }^{2}$ Ovarian Cancer \\ Research, Department of Biomedicine, \\ University Hospital Basel, University \\ of Basel, Basel, Switzerland; \\ ${ }^{3}$ Department of Gynecology, \\ University Hospital Zurich, Zürich, \\ Switzerland; ${ }^{4}$ Gynecological Cancer \\ Centre, Royal Hospital for Women, \\ School of Women's and Children's \\ Health, University of New South \\ Wales, Randwick, NSW, Australia
}

Correspondence: Céline Montavon Sartorius

Women Hospital, Gynecological Oncology, University Hospital Basel, Spitalstrasse 2I, Basel 403I, Switzerland Tel +4I 612659099

Fax +4I 612659199

Email celine.montavon@usb.ch
Purpose: The stage of disease is one of the strongest prognostic factors in epithelial ovarian cancer. The International Federation of Gynecology and Obstetrics (FIGO) classification was revised in 2013; stage IC was subdivided into IC1 (intraoperative surgical spill), IC2 (capsule rupture before surgery or tumor on surface), and IC3 (positive peritoneal washing or ascites). Our aim was to compare the outcome of patients in the new FIGO stage I subgroups, as this might influence adjuvant therapy decisions.

Patients and methods: Patient databases of three gynecological oncology centers were retrospectively analyzed. Patients with FIGO stage I ovarian cancers were restaged according to the revised classification, based on operative and pathological reports, and determined patient outcomes.

Results: We analyzed 128 patients with ovarian cancers. In FIGO IA, we found $11.3 \%$ recurrences and $4.2 \%$ deaths. In FIGO IC, $21.8 \%$ of the patients recurred and $7.3 \%$ died. There was a trend toward a shorter time to recurrence when comparing IA to IC $(P=0.076)$. Within all new subgroups of FIGO IC, there was no difference in time to recurrence $(P=0.59)$. There was also no significant difference in survival when FIGO IA was compared to FIGO IC in comparison with the new individual classifications (IA to IC, IA to IC1 , 2, or 3; $P=0.60, P=0.15, P=0.61, P=0.66$, respectively) or within the different subgroups $(P=0.56)$. Platinum-based chemotherapy was given to the majority $(82.6 \%, \mathrm{n}=38 / 46)$ of the FIGO IC patients compared to $30.9 \%$ in FIGO IA $(n=17 / 55)$. There was no significant difference within the new subgroups of FIGO IC $(P=0.88)$. Conclusion: In our retrospective analysis, the new FIGO staging of IC ovarian cancers did not predict prognosis, but the use of adjuvant chemotherapy in $82.6 \%$ of the stage IC patients may have biased the outcome.

Keywords: ovarian neoplasm, cancer staging, survival, recurrence

\section{Introduction}

Epithelial ovarian cancer is the fifth most common cancer among women. In Europe, about 42,000 women are diagnosed with ovarian cancer and 29,200 women die from this disease each year. ${ }^{1}$ About one quarter of patients present with early ovarian cancer (International Federation of Gynecology and Obstetrics [FIGO] stages I and II), where the overall 5-year survival is reported to be between $80 \%$ and $90 \%$. $^{2}$

For ovarian cancer, FIGO staging system is commonly used, and, especially in early ovarian cancer, surgical and pathological staging is crucial. The purpose of a unified staging system is as follows: first, to develop an accurate and universal terminology to describe the extent of disease; second, to allocate patients to prognostic subgroups; and third, to compare treatment efficacy and survival outcomes between centers as part of 
clinical trials and research. ${ }^{3}$ The stage should reflect the extent of the metastatic tumor burden and, most importantly, prognosis.

Along with histological type, grade, and residual disease, stage is a powerful prognostic factor. Up to one-third of patients with apparent early ovarian cancer are upstaged when proper surgical staging is performed, and surgical staging is an independent prognostic factor for survival. ${ }^{4}$ Complete surgical staging should include peritoneal washings, hysterectomy, bilateral salpingo-oophorectomy, omentectomy, multiple peritoneal biopsies, and pelvic and para-aortic lymphadenectomy. Bilateral ovarian lesions $(P<0.001)$, positive cytology $(P=0.012)$, presence of ascites $(P=0.002)$, highgrade histology $(P<0.001)$, and serous histology $(P=0.001)$ are risk factors for lymph node involvement, which should be considered in counseling patients for restaging after unexpected findings of malignancy and incomplete surgery. ${ }^{5}$

The 1988 FIGO classification for cancer of the ovary was revised in 2013 after 24 years of use (Table 1). ${ }^{6}$ The objective of this study was to evaluate the clinical impact of the new classification system on early-stage ovarian cancer, particularly the new subgroups of stage IC.

\section{Patients and methods}

We retrospectively reviewed all cases of FIGO stage I epithelial ovarian and fallopian tube cancers from the databases of three major gynecological oncology centers from two countries (Switzerland and Australia): The Women's Hospital, University Hospital of Basel, Switzerland, The Women's Hospital, University Hospital of Zurich, Switzerland, and The Royal Hospital for Women in Sydney, Australia, in the time frame between 1992 and 2015. Our final cohort consisted of 128 cases of FIGO I ovarian cancer $(n=124)$ and FIGO I fallopian tubal cancer $(n=4)$.

Demographical, surgical, histological, treatment, and outcome data of these patients were analyzed. We restaged the patients according to the new FIGO guidelines from a homogenous group of IC to IC1, IC2, and IC3. Restaging to IC1 was based on operative reports confirming intraoperative rupture. Restaging to IC2 and IC3 was based on both operative and pathology reports confirming capsule rupture before surgery, tumor seeds on the surface of the ovary, or positive peritoneal washing cytology.

Complete surgical staging included peritoneal washings, hysterectomy, bilateral salpingo-oophorectomy, pelvic and para-aortic lymphadenectomy, infracolic omentectomy, and multiple peritoneal biopsies.

After surgery, the indication for adjuvant chemotherapy was discussed at an interdisciplinary tumor board for all cases. Adjuvant chemotherapy consisted of platinum-based chemotherapy. No chemotherapy was given for cases of grade 1, FIGO stages IA and IB tumors. Patients were followed up every 3 months for the first 2 years, half-yearly for up to 5 years, and then annually until 10 years. Patients were monitored clinically, biochemically by tumor markers, and radiologically (baseline CT directly after surgery, further CT indicated by clinical and/or biochemical suspicion of relapse).

\section{Statistical methods}

Relapse-free survival (RFS) and disease-specific survival (DSS) were displayed as Kaplan-Meier curves with corresponding $P$-values of the log-rank test. Additionally, number at risk and number of events were reported for each group

Table I Details of the FIGO classification for ovarian and fallopian tube(s) cancers: old I 988 vs new 2013 FIGO staging

\begin{tabular}{|c|c|c|c|c|}
\hline & & Old - I988 FIGO stage & & New - 2013 FIGO stage \\
\hline \multirow[t]{6}{*}{ Stage I } & IA & $\begin{array}{l}\text { Tumor limited to one ovary, capsule } \\
\text { intact, no tumor on ovarian surface, and } \\
\text { negative washings/ascites }\end{array}$ & IA & $\begin{array}{l}\text { Tumor limited to one ovary or fallopian tube, } \\
\text { capsule intact, no tumor on surface, and } \\
\text { negative washings/ascites }\end{array}$ \\
\hline & IB & $\begin{array}{l}\text { Tumor involves both ovaries, capsule } \\
\text { intact, no tumor on ovarian surface, and } \\
\text { negative washings/ascites }\end{array}$ & IB & $\begin{array}{l}\text { Tumor limited to both ovaries or fallopian } \\
\text { tubes, capsule intact, no tumor on surface, } \\
\text { and negative washings/ascites }\end{array}$ \\
\hline & \multirow[t]{4}{*}{ IC } & \multirow{4}{*}{$\begin{array}{l}\text { Tumor limited to ovaries with any of } \\
\text { the following: capsule rupture, tumor on } \\
\text { ovarian surface, or positive washing/ascites }\end{array}$} & IC & $\begin{array}{l}\text { Tumor limited to one or both ovaries or } \\
\text { fallopian tube }\end{array}$ \\
\hline & & & $\mathrm{ICl}$ & - With surgical spill \\
\hline & & & IC2 & $\begin{array}{l}\text { With capsule rupture before surgery or } \\
\text { tumor on ovarian or fallopian tube surface }\end{array}$ \\
\hline & & & IC3 & $\begin{array}{l}\text { With malignant cells in the ascites or } \\
\text { peritoneal washings }\end{array}$ \\
\hline
\end{tabular}

Abbreviation: FIGO, International Federation of Gynecology and Obstetrics. 
separately. Comparisons of RFS and DSS within individual subgroups were done using the log-rank test.

In order to compare chemotherapy-adjusted RFS between study groups, Cox regression was performed. Results are reported as HR with corresponding 95\% CI and $P$-values. DSS comparison was not done because of low number of events.

In the case of ordinal or metric variables, median and interquartile range with corresponding Wilcoxon tests were calculated. In the case of categorical variables, counts and percentages were reported, and Fisher's exact test was performed. A $P$-value of $<0.05$ was considered as significant. All evaluations were performed using the statistical software $\mathrm{R}$, version 3.2.1.

The study was approved by the respective medical ethics committees: the Ethikkommission Nordwest- und Zentralschweiz (EKNZ BASEC 2016-01011) and the Hunter New England Human Research Ethics Committee (HNEHREC 16/04/20/5.06). Patient consent to review their medical records was not required by the ethics committees for the time frame of this study (patient database from 1992 up to 2015). The study was retrospective and constituted no harm for patients. Patient data confidentiality has been protected, and the medical data were used anonymously. As required by the ethics committees, this study has been conducted in compliance with the protocol, the current version of the Declaration of Helsinki, the ICH-GCP or ISO EN 14155 (as far as applicable) as well as all national legal and regulatory requirements.

\section{Results}

Our databases collectively consisted of data of 2,957 patients with gynecological tumors diagnosed in the time frame between 1992 and 2015. Of these, we identified 131 peritoneal cancers, 1,020 ovarian cancers, and 48 tubal cancers. We excluded all cases other than epithelial ovarian and tubal cancers, FIGO stage I. Patients with synchronous tumors of the ovary/tube and endometrial cancer $(n=12)$ were also excluded. Of the epithelial ovarian and tubal cancer cases, 206 were determined to be of FIGO stage I. After excluding borderline ovarian tumors $(n=78)$, we were left with a final cohort of $n=128$ consisting of FIGO I ovarian cancer $(n=124)$ and FIGO I fallopian tubal cancer $(n=4)$. The histology of these cases was as follows: serous $28.1 \%(n=37)$, endometrioid $25.8 \%(n=33)$, mucinous $25 \%(n=32)$, clear cell $12.5 \%$ $(\mathrm{n}=16)$, mixed (including carcinosarcoma) $6.9 \%(\mathrm{n}=9)$, and transitional cell $1.6 \%(\mathrm{n}=2)$. Tumor grade $(\mathrm{n}=103)$ was as follows: grade $1(n=37) 35.9 \%$, grade $2(n=26) 25.2 \%$, and grade $3(n=40) 38.8 \%$. FIGO stages at diagnosis consisted of IA $(n=71) 55.5 \%$, IB $(n=2) 1.5 \%$, and IC $(n=55) 43 \%$. Upon restaging, the FIGO IC group consisted of FIGO IC1 $(n=14)$ $10.9 \%$, IC2 $(n=11) 8.6 \%$, and IC3 $(n=30) 23.5 \%$. The mean age was 56.6 years $(\mathrm{SD}=15.1)$. Mean length of follow-up was 59.4 months (range 0-258 months) (Table 2).

The outcome has been measured on the entire cohort $(n=128)$. We performed a survival analysis and defined RFS as the time from the initial treatment (surgery) to relapse or the last follow-up visit. We observed a total of 20 recurrences, reported by stage: IA $n=8 / 68$, IB $n=0 / 1, I C 1 n=2 / 14$, IC 2 $n=3 / 11$, and IC $3 n=7 / 27$. The repartition of the recurrences by histological type was as follows: serous $30.0 \%(n=6)$, endometrioid $30.0 \%(n=6)$, mucinous $10.0 \%(n=2)$, clear cell $10.0 \%(n=2)$, mixed (including carcinosarcoma) $20 \%(n=4)$, and transitional cell $10.0 \%(n=2)$. Patients with recurrence and their characteristics are listed in Table 3. DSS was defined as the time from the initial treatment to death due to initial diagnosis. We reported six deaths in our cohort, namely in the group of FIGO stages IA $n=3 / 68$, IB $n=0 / 1$, IC $1 n=1 / 14$, IC2 $n=0 / 11$, and IC3 n=2/27. Kaplan-Meier curves for the RFS and DSS are shown in Figures 1-4.

To define the staging quality of our cohort, we analyzed the surgical reports of each patient. In 12 patients we had insufficient data to evaluate the extent of staging. Therefore, these patients were excluded from this part of the analysis. Complete surgical staging, including systematic pelvic and paraaortic lymphadenectomy, was performed in 72 patients $(n=116$, $62.1 \%$ ). Systematic intraperitoneal staging, however, without lymphadenectomy was performed in 34 patients $(29.3 \%)$. A further 10 patients $(8.6 \%)$ were incompletely staged; the reason in most cases reflected the patient's choice, and in one case it was because of fertility preservation. Patients were not excluded from the other analysis based on their staging status.

\section{IA vs IC}

Of the FIGO IA cases, there were eight recurrences $(11.3 \%)$ and three deaths (4.2\%). The 5-year RFS was $87 \%$, and the 5 -year DSS was 98\%. For all subgroups of IC, there were 12 recurrences $(21.8 \%)$ and four deaths $(7.3 \%)$, the 5 -year RFS was $67 \%$, and the 5 -year DSS was $98 \%$. There was no significant difference in RFS or DSS $(P=0.076$ and 0.60 , respectively) between FIGO stages IA and IC as measured by the log-rank test.

\section{ICI vs IC2 vs IC3}

When comparing the new subgroups of FIGO IC, the 5-year RFS was 62\% (IC1), 78\% (IC2), and 59\% (IC3) $(P=0.59)$. 
Table 2 Patient characteristics

\begin{tabular}{|c|c|c|c|c|c|c|c|c|}
\hline \multirow{2}{*}{$\begin{array}{l}\text { Baseline } \\
\text { characteristics }\end{array}$} & All & IA & IB & ICI & $1 C 2$ & IC3 & \multirow[t]{2}{*}{$P$-value } & \multirow[t]{2}{*}{$\mathbf{N}$} \\
\hline & $n=128$ & $n=7 \mid$ & $n=2$ & $n=14$ & $n=I I$ & $n=30$ & & \\
\hline $\begin{array}{l}\text { Primary site } \\
\text { - Ovary } \\
\text { - Fallopian tube }\end{array}$ & $\begin{array}{l}124(96.9 \%) \\
4(3.12 \%)\end{array}$ & $\begin{array}{l}7 I(100 \%) \\
0\end{array}$ & $\begin{array}{l}2(100 \%) \\
0\end{array}$ & $\begin{array}{l}\text { I3 (92.9\%) } \\
\text { I (7.I4\%) }\end{array}$ & $\begin{array}{l}10(90.9 \%) \\
1(9.09 \%)\end{array}$ & $\begin{array}{l}28(93.3 \%) \\
2(6.67 \%)\end{array}$ & $0.07 I$ & 128 \\
\hline $\begin{array}{l}\text { Histology } \\
\text { - Serous } \\
\text { - Clear cell } \\
\text { - Endometrioid } \\
\text { - Mucinous } \\
\text { - Transitional } \\
\text { - MMMT, mixed }\end{array}$ & $\begin{array}{l}36(28.1 \%) \\
16(12.5 \%) \\
33(25.8 \%) \\
32(25.0 \%) \\
2(1.56 \%) \\
9(6.98 \%)\end{array}$ & $\begin{array}{l}14(19.7 \%) \\
13(18.3 \%) \\
18(25.4 \%) \\
22(31.0 \%) \\
\text { I (1.4I\%) } \\
3(4.17 \%)\end{array}$ & $\begin{array}{l}\text { I (50.0\%) } \\
0 \\
\text { I (50.0\%) } \\
0 \\
0 \\
0\end{array}$ & $\begin{array}{l}3 \text { (2I.4\%) } \\
\text { I (7.I4\%) } \\
6 \text { (42.9\%) } \\
4 \text { (28.6\%) } \\
0 \\
0\end{array}$ & $\begin{array}{l}4(36.4 \%) \\
0 \\
3(27.3 \%) \\
2(18.2 \%) \\
0 \\
2(18.2 \%)\end{array}$ & $\begin{array}{l}14(46.7 \%) \\
2(6.67 \%) \\
5(16.7 \%) \\
4(13.3 \%) \\
1(3.33 \%) \\
4(13.33 \%)\end{array}$ & 0.300 & 128 \\
\hline $\begin{array}{l}\text { Grade } \\
\text { - } \mathrm{GI} \\
\text { - } \mathrm{G} 2 \\
\text { - } \mathrm{G} 3 \\
\text { - } \text { Missing }\end{array}$ & $\begin{array}{l}37(35.9 \%) \\
26(25.2 \%) \\
40(38.8 \%) \\
25\end{array}$ & $\begin{array}{l}27(49.1 \%) \\
14(25.5 \%) \\
14(25.5 \%)\end{array}$ & $\begin{array}{l}0 \\
0 \\
I(100 \%)\end{array}$ & $\begin{array}{l}5(45.5 \%) \\
2(18.2 \%) \\
4(36.4 \%)\end{array}$ & $\begin{array}{l}2(22.2 \%) \\
3(33.3 \%) \\
4(44.4 \%)\end{array}$ & $\begin{array}{l}3(11.1 \%) \\
7(25.9 \%) \\
17(63.0 \%)\end{array}$ & 0.011 & 103 \\
\hline $\begin{array}{l}\text { Staging }^{\mathbf{a}} \\
\text { - Complete } \\
\text { - Incomplete } \\
\text { - Missing }\end{array}$ & $\begin{array}{l}72(62.1 \%) \\
44(37.9 \%) \\
12\end{array}$ & $\begin{array}{l}39(60.9 \% \\
25(39.1 \%)\end{array}$ & $\begin{array}{l}0 \\
2(100 \%)\end{array}$ & $\begin{array}{l}8(57.1 \%) \\
6(42.9 \%)\end{array}$ & $\begin{array}{l}6(66.7 \%) \\
3(33.3 \%)\end{array}$ & $\begin{array}{l}19(70.4 \%) \\
8(29.6 \%)\end{array}$ & 0.426 & 116 \\
\hline $\begin{array}{l}\text { Chemotherapy }^{a} \\
\text { - No } \\
\text { - Yes } \\
\text { - Missing }\end{array}$ & $\begin{array}{l}46(45.1 \%) \\
56(54.9 \%) \\
26\end{array}$ & $\begin{array}{l}38(69.1 \%) \\
17(30.9 \%)\end{array}$ & $\begin{array}{l}0 \\
\text { I (100\%) }\end{array}$ & $\begin{array}{l}2(18.2 \%) \\
9(81.8 \%)\end{array}$ & $\begin{array}{l}2(22.2 \%) \\
7(77.8 \%)\end{array}$ & $\begin{array}{l}4(15.4 \%) \\
22(84.6 \%)\end{array}$ & $>0.001$ & 102 \\
\hline $\begin{array}{l}\text { Follow-up months, } \\
\text { median (IQR) } \\
\text { Missing }\end{array}$ & $\begin{array}{l}37.0 \\
{[12.5 ; 92.5]} \\
5\end{array}$ & $\begin{array}{l}35.0 \\
{[12.0 ; 111]}\end{array}$ & $\begin{array}{l}19.0 \\
{[19.0 ; 19.0]}\end{array}$ & $\begin{array}{l}35.5 \\
{[12.5 ; 66.5]}\end{array}$ & $\begin{array}{l}60.0 \\
{[36.0 ; 92.5]}\end{array}$ & $\begin{array}{l}27.0 \\
{[13.0 ; 68.2]}\end{array}$ & 0.803 & 123 \\
\hline Age, years & $\begin{array}{l}56.0 \\
{[45.0 ; 68.0]}\end{array}$ & $\begin{array}{l}60.0 \\
{[47.5 ; 67.5]}\end{array}$ & $\begin{array}{l}67.0 \\
{[57.5 ; 76.5]}\end{array}$ & $\begin{array}{l}46.5 \\
{[41.5 ; 56.0]}\end{array}$ & $\begin{array}{l}59.0 \\
{[42.0 ; 69.5]}\end{array}$ & $\begin{array}{l}55.0 \\
{[45.5 ; 68.8]}\end{array}$ & 0.206 & 128 \\
\hline
\end{tabular}

Notes: aPercentages calculated excluding missing values from denominator. N, number available for each variable; Missing, information on patient was missing; Chemotherapy, adjuvant platinum-based chemotherapy.

Abbreviations: IQR, interquartile range; MMMT, malignant mixed Mullerian tumor or carcinosarcoma; n, number.

The 5-year DSS was $100 \%$ (IC1), $100 \%$ (IC2), and $96 \%$ (IC3) $(P=0.56)$.

\section{IA vs ICI, IC2, and IC3, respectively}

There was no significant difference in RFS or DSS when comparing FIGO IA to IC1, IC2, or IC3 individually (RFS: $P=0.47, P=0.23 ; P=0.07$, respectively, and DSS: $P=0.14$; $P=0.61, P=0.66$, respectively). The FIGO group IB could not be compared to the other groups because the study had only one patient with that stage.

\section{Sub-analysis}

We performed a sub-analysis by grade and histological subtype; however, neither of these showed any significant differences in RFS or DSS with the log-rank test. For high grade (G3) tumors ( $n=40)$, the stages were as follows: FIGO
IA $n=14$, IB $n=1$, IC $1 n=4$, IC $2 n=4$, and IC $3 n=17$. The survival analysis showed no significant difference between all groups, RFS $P=0.68$ and DSS $P=0.9$. Within the serous histology ( $n=37$; FIGO IA $n=14$, IB $n=1$, IC1 n=3, IC2 n=4, and IC3 $n=14)$, there was no significant difference for the RFS $(P=0.62)$ as well as for the DSS $(P=0.63)$

\section{Chemotherapy}

In the FIGO stage IA group, platinum-based chemotherapy was administered in $30.9 \%(n=17)$ of the patients, while in FIGO IC $82.6 \%(n=38)$ received this treatment. Within the FIGO IC group, there was no difference in chemotherapy administration rates between the new sub-classifications; chemotherapy for FIGO IC1, IC2, and IC3 was administered in $81.8 \%(\mathrm{n}=9), 77.8 \%(\mathrm{n}=7)$, and $84.6 \%(\mathrm{n}=22)$ of cases, respectively. As expected, the adjuvant therapeutic proce- 
Table 3 Patients with recurrence and FIGO I stage disease

\begin{tabular}{|c|c|}
\hline \multirow[t]{2}{*}{ Baseline characteristics } & Recurrence \\
\hline & $\mathbf{n}=\mathbf{2 0}$ \\
\hline $\begin{array}{l}\text { Primary site } \\
\text { - Ovary } \\
\text { - Fallopian tube }\end{array}$ & $\begin{array}{l}19(95 \%) \\
\text { I (5\%) }\end{array}$ \\
\hline $\begin{array}{l}\text { Histology } \\
\text { - Serous } \\
\text { - Clear cell } \\
\text { - Endometrioid } \\
\text { - Mucinous } \\
\text { - Transitional } \\
\text { - MMMT, mixed }\end{array}$ & $\begin{array}{l}6(30.0 \%) \\
2(10.0 \%) \\
6(30.0 \%) \\
2(10.0 \%) \\
0 \\
4(20.0 \%)\end{array}$ \\
\hline $\begin{array}{l}\text { FIGO stage } \\
\text { - } \mathrm{IA} \\
\text { - } \mathrm{IB} \\
\text { - } \mathrm{ICI} \\
\text { - } \mathrm{IC} 2 \\
\text { - } \mathrm{IC} 3\end{array}$ & $\begin{array}{l}8(40.0 \%) \\
0 \\
2(10.0 \%) \\
3(15.0 \%) \\
7(35.0 \%)\end{array}$ \\
\hline $\begin{array}{l}\text { Grade }^{\mathrm{a}} \\
\text { - } \mathrm{Gl} \\
\text { - } \mathrm{G} 2 \\
\text { - } \mathrm{G} 3 \\
\text { - } \text { Missing }\end{array}$ & $\begin{array}{l}4(25.0 \%) \\
5 \text { (3I.2\%) } \\
7 \text { (43.8\%) } \\
4\end{array}$ \\
\hline $\begin{array}{l}\text { Staging } \\
\text { - } \quad \text { Complete } \\
\text { - Incomplete } \\
\text { - } \text { Missing }\end{array}$ & $\begin{array}{l}10(66.7 \%) \\
5(33.3 \%) \\
5\end{array}$ \\
\hline $\begin{array}{l}\text { Chemotherapya } \\
\text { - No } \\
\text { - Yes } \\
\text { - Missing }\end{array}$ & $\begin{array}{l}3(20.0 \%) \\
12(80.0 \%) \\
5\end{array}$ \\
\hline Follow-up months, median (IQR) & $46.0[7.00 ; 174]$ \\
\hline Age, years & $61.0[23.0 ; 85.0]$ \\
\hline
\end{tabular}

Notes: ${ }^{\text {P}}$ ercentages calculated excluding missing values from denominator. Missing, information on patient was missing; Chemotherapy, adjuvant platinum-based chemotherapy.

Abbreviations: FIGO, International Federation of Gynecology and Obstetrics; IQR, interquartile range; MMMT, malignant mixed Mullerian tumor or carcinosarcoma; $n$, number.

dure differs significantly between the FIGO IA, IB, and IC $(P<0.001)$, but not within the new FIGO IC subclassification $(P=0.88)$.

We also performed a multivariate regression analysis to compare chemotherapy-adjusted RFS between study groups. There was no significant HR. The comparison was not performed for DSS because of very small number of events (deaths $\mathrm{n}=6$ ). The results are reported in Table 4 .

No additional multivariate Cox model (adjusting for other study parameters) was done as the inclusion of several covariates in a study with relatively small event rates can lead to spurious results. ${ }^{7}$

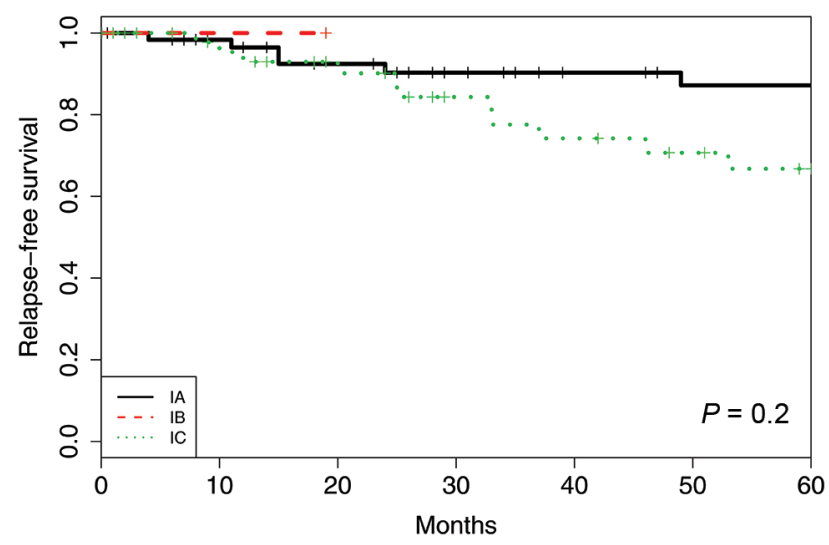

Figure I Relapse-free survival using the old FIGO classification system for the cohort of I 28 patients with ovarian and tubal cancers in FIGO I stage.

Abbreviation: FIGO, International Federation of Gynecology and Obstetrics.

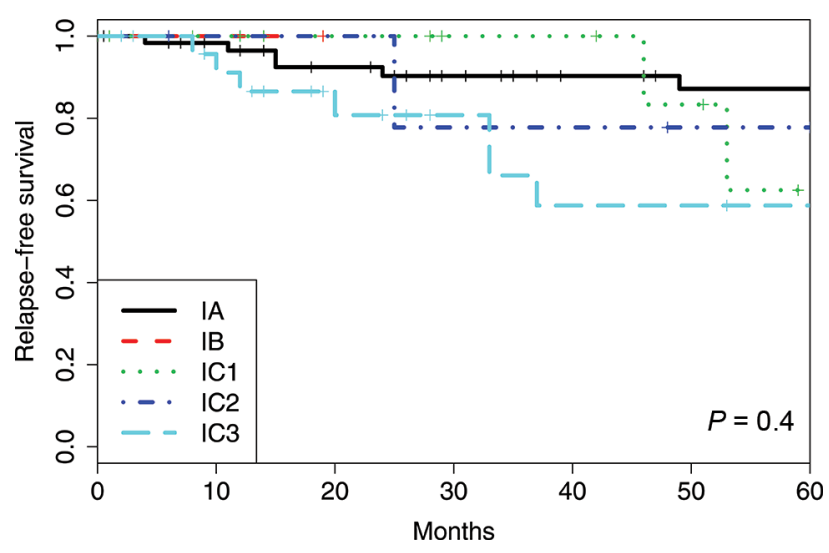

Figure 2 Relapse-free survival using the new FIGO classification system for the cohort of 128 patients with ovarian and tubal cancers in FIGO I stage.

Abbreviation: FIGO, International Federation of Gynecology and Obstetrics.

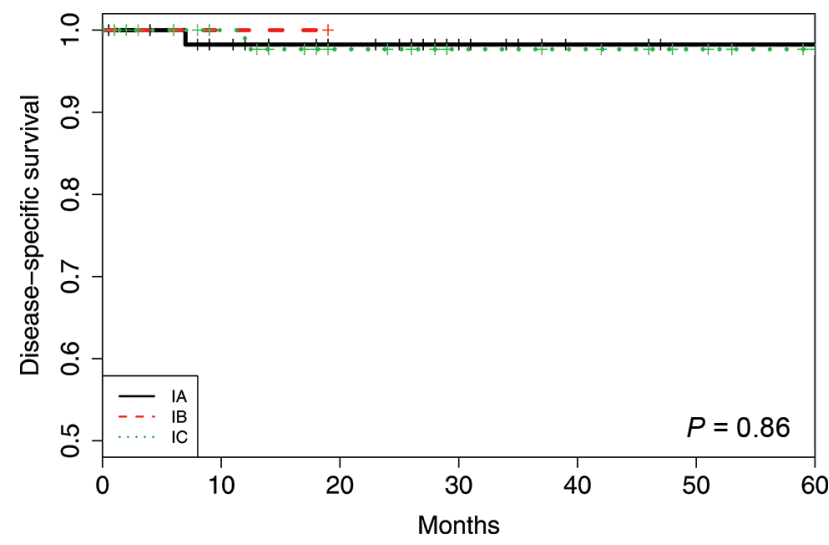

Figure 3 Disease-specific survival using the old FIGO classification system for the cohort of I 28 patients with ovarian and tubal cancers in FIGO I stage.

Abbreviation: FIGO, International Federation of Gynecology and Obstetrics. 


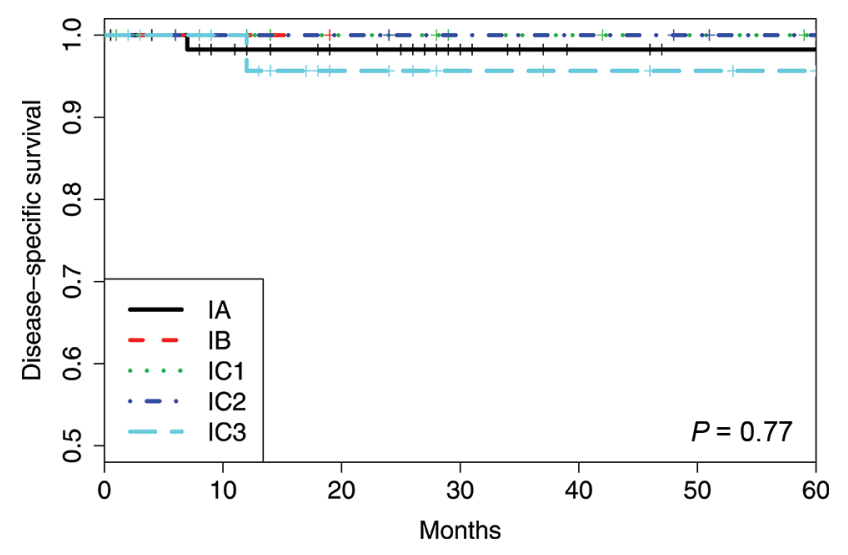

Figure 4 Disease-specific survival using the new FIGO classification system for the cohort of 128 patients with ovarian and tubal cancers in FIGO I stage.

Abbreviation: FIGO, International Federation of Gynecology and Obstetrics.

Table 4 Subgroups comparison reported as HR adjusted for chemotherapy, with lower and upper $95 \%$ confidence limits

\begin{tabular}{|l|l|l|l|l|}
\hline Comparison & HR & LCL & UCL & $P$-value \\
\hline IA vs ICI & 1.83 & 0.19 & 18.02 & 0.604 \\
IA vs IC2 & 0.98 & 0.17 & 5.65 & $0.98 I$ \\
IA vs IC3 & 0.56 & 0.14 & 2.18 & 0.404 \\
ICI vs IC2 & 0.53 & 0.05 & 5.96 & $0.6 I I$ \\
ICI vs IC3 & $0.3 I$ & 0.04 & 2.52 & 0.273 \\
IC2 vs IC3 & 0.57 & 0.12 & 2.8 & 0.492 \\
\hline
\end{tabular}

Abbreviations: LCL, lower confidence limit; UCL, upper confidence limit.

\section{Discussion FIGO staging}

With the updated 2013 FIGO staging for ovarian, fallopian tube, and primary peritoneal cancers, the quantitative number of substages changed from 10 to $14 .^{6}$ Staging underwent a major revision and became more demanding as the same number of patients are now distributed in a larger number of categories, thereby decreasing patient numbers per substage. The consequence of this is that it becomes more difficult to show a real difference between the different subgroups.

Our study focused on the impact of the stratification of FIGO IC, which has been divided into the following three further categories: IC1, surgical spill intraoperatively, IC2, capsular ruptured before surgery or tumor on ovarian or fallopian tube surface, and IC3, malignant cells in the ascites or peritoneal washings. Intuitively, one would think that an iatrogenic capsule rupture, following which the peritoneal cavity can be washed with water, should not have the same clinical impact as an ovarian cancer, which has already spontaneously spread, as demonstrated by positive peritoneal washings.
In our analysis on 128 ovarian cancers, the revised FIGO system for stage I did not add prognostic information. We could not confirm that surgical spill would have a similar outcome as a FIGO IA stage. FIGO IC showed more recurrences and deaths compared to FIGO IA $(21.8 \%$ vs $11.3 \%$ recurrences, $7.3 \%$ vs $4.2 \%$ deaths). There was a trend toward a shorter time to recurrence when comparing IA to IC $(P=0.076)$, however, without impact on survival. There was also no significant difference in survival when FIGO IA was compared to FIGO IC in comparison with the new individual classifications or within the different subgroups.

Nevertheless, the particularity of our cohort is a substantially higher percentage of completely and correctly staged patients $(62.1 \%)$ compared to other studies. A complete surgical staging procedure in early-stage ovarian and fallopian tube cancers should include hysterectomy with bilateral salpingo-oophorectomy, pelvic and paraaortic lymph node dissection as well as omentectomy, peritoneal washings, peritoneal biopsies, and biopsies of any suspicious nodules. ${ }^{8}$ Incomplete staging could lead to incorrect staging.

Clear prognostic differences between different subgroups of FIGO I should lead to adjuvant treatment recommendations, particularly with respect to adjuvant platinum-based chemotherapy. Only one study, facilitating a nationwide register, has shown that FIGO IA and IC1 (surgical spill intraoperatively) have a similar prognosis. ${ }^{9}$ Rosendahl et al published data on 4,036 ovarian cancer patients who were assigned to the new stages ${ }^{9}$ and illustrated similar survival characteristics in two groups: FIGO IA $(n=464)$ with IC1 $(n=143)$ (5-year overall survival [OS], 87\%), and FIGO IB $(\mathrm{n}=51)$ with IC2 $(\mathrm{n}=195)$ and with IC3 $(\mathrm{n}=140)$ (5-year OS, $75 \%-80 \%)$. This suggests that surgical spillage has little or no significance, which is a relevant finding for gynecological oncologists operating on such patients. One significant problem in this cohort, however, is the high percentage of incompletely staged patients (in stages IA-IIB lymphadenectomy was only performed in $30 \%-43 \%$ and omentectomy in $81 \%-93 \%$ of cases). ${ }^{9}$ Patients with positive lymph nodes, occult peritoneal, or omental metastases would have been upstaged and could have modified the results found on the survival.

During the revision process of the new FIGO classification, Suh et al analyzed 254 cases with FIGO stage I disease. The breakdown by subtype was as follows: IA $n=128$, IB $\mathrm{n}=7$, and IC $\mathrm{n}=119$ (IC1: 39; IC2: 27, and IC3: 53). The 5-year OS rates of patients with stage IA were $93.5 \%$ and with substages IC1, IC2, and IC3 were $92.0 \%, 85.0 \%$, and 
$71.0 \%$, respectively $(P=0.004)$. These data suggested that patients with surgical spillage had the best outcome which seems somehow difficult to anticipate. ${ }^{10}$

Paik et al investigated the influence of the revised FIGO staging system on 200 cases of FIGO stage I disease. The subgroups were as follows: IA $n=96$, IB $n=6$, and IC $n=98$ (IC1: 9; IC2: 57; and IC3: 32). ${ }^{11}$ They were unable to show a significant difference between the groups and subgroups in the new classification using univariate analysis. In multivariate analysis, FIGO stage IC3 was shown to have a significantly worse progression-free survival (PFS) $(P=0.011)$, but not on OS. This may be due to a lack of statistical power because of low numbers. ${ }^{11}$

The survival outcome in our cohort was better compared to the prior reports with a 5-year DSS in FIGO I of 96\%-100\%. We reported both the 5-year RFS and 5-year DSS as FIGO IA $87 \%$ and $98 \%$ and FIGO IC $67 \%$ and $98 \%$, respectively. Within the FIGO IC subgroup, the 5-year RFS was $62 \%$ (IC1), 78\% (IC2), and 59\% (IC3), and the 5-year DSS was 100\% (IC1), 100\% (IC2), and 96\% (IC3).

\section{Role of classification systems}

Recent findings in ovarian cancer research underline a new approach to this heterogeneous disease due to different groups of tumors with different characteristics, phenotypes, origins, and biology. ${ }^{12}$ However, the aim of a classification system for malignancy should be to assign patients to groups that allow meaningful stratification for prognosis. It should also offer easy communication with standard terminology among physicians, allowing comparison between centers and consistency for clinical trials. Finally, it should be easy to use and reproducible. .,6,13,14 $^{2}$

The tumor biology is not integrated into the actual classification, although low and high grade, ovarian and fallopian tube cancers are different molecular entities and behave in different ways. We, therefore, performed a subgroup analysis for high and low grade and for serous and non-serous ovarian and tubal cancers. There was no survival difference between the groups. However, the cancer heterogeneity could be the reason why Paik et al have a significant result only after multivariate analysis excluding other prognostic cofounders, ${ }^{11}$ and why the literature shows such divergent results. ${ }^{9,10,15}$ The new FIGO classification appears to be generally more difficult to apply in daily practice, and research analysis is more difficult due to a smaller number of cases in each subgroup. The retrospective character of the trials and their selection bias must also be taken into account.

\section{Incomplete staging}

Incomplete staging is a major source of bias. The strength of this study is the low level of incompletely staged patients $(37.9 \%)$. A systematic intraperitoneal staging with peritoneal washings, hysterectomy, bilateral salpingooophorectomy, omentectomy, and multiple biopsies was performed in $91.4 \%$ of the cases, including $62.1 \%$ of the completely staged patients with systematic pelvic and paraaortic lymphadenectomy. Trimbos et al included 40 centers in nine European countries with a precise protocol as part of a randomized Phase III trial (ACTION Trial) ${ }^{16}$ to study the impact of adjuvant chemotherapy and surgical staging in early-stage ovarian carcinoma. However, even in this randomized trial in which comprehensive surgical staging was a requirement in the study protocol, the majority of patients $(66 \%)$ were incompletely staged with one or more staging procedures omitted. ${ }^{17}$

The high level of fully staged patients in this study is explained by the fact that all operations were performed within gynecological cancer centers, with trained gynecological oncology surgeons available to perform the staging procedure. If the cancer diagnosis had been made unexpectedly and the staging was insufficient, a second operation to complete the staging was strongly recommended. In $29.3 \%$ of the cases, there was no staging lymphadenectomy, usually due to patient's choice. Paik et al showed a significant prognostic impact on PFS for stage IC3 in the multivariate analysis only by adjusting age, cell type, grade, and surgical staging methods (complete staging vs incomplete staging), which underlines the impact of staging on survival.

To further illustrate the importance of complete surgical staging, it has recently been shown that in patients with stages I-IIA ovarian cancer who had $\geq 10$ lymph nodes removed, there was no further benefit from chemotherapy. This was also true for a subgroup of patients with high-risk features (stages IC and IIA and/or tumor grade 3 and/or clear cell histology). ${ }^{18}$

\section{Chemotherapy effect}

Another important potential bias comes from chemotherapy. Two parallel prospective randomized trials have addressed the issue of adjuvant chemotherapy in patients with earlystage ovarian cancer ${ }^{16,19}$ but only one, the ACTION study, required complete surgical staging. The Adjuvant Chemotherapy in Ovarian Neoplasm (ACTION) trial study is a prospective randomized Phase III trial that tests the efficacy of adjuvant chemotherapy in 448 patients from 40 centers 
in nine European countries with early-stage ovarian cancer, with emphasis on the extent of surgical staging. Patients were randomized in two arms after surgery, namely 1) adjuvant platinum-based chemotherapy $(n=224)$ or 2$)$ observation only $(n=224) .{ }^{16}$ After a median follow-up of 5.5 years, there was no significant difference in OS between the two arms, in contrast to the RFS which was significantly improved in the adjuvant chemotherapy $\operatorname{arm}(\mathrm{HR}=0.63,95 \% \mathrm{CI}=0.43-0.92$, $P=0.10)$. One-third of patients were optimally staged, but two-third were not. The long-term analysis of the ACTION Trial $^{20}$ after a median follow-up of 10.1 years demonstrated that OS after optimal surgical staging was improved, even among patients who received adjuvant chemotherapy (HR $=1.89,95 \% \mathrm{CI}=0.99-3.60, P=0.05)$, however, the benefit from adjuvant chemotherapy in early-stage ovarian cancer was limited to patients with nonoptimal surgical staging.

Patients $(n=477)$ in the International Collaborative Ovarian Neoplasm trial 1 (ICON1) study were recruited in 84 centers in five countries and were randomized in one group which received adjuvant chemotherapy immediately after surgery $(n=241)$ or in another group without chemotherapy until clinically indicated $(n=236) .{ }^{19}$ A complete surgical staging was not required, the staging procedure was not recorded, so that the proportion of optimally staged patient remains unknown. With a median follow-up of 4 years, ICON1 demonstrated a significant improvement in $\mathrm{RFS}(\mathrm{HR}=0.66,95 \% \mathrm{CI}$ $=0.45-0.97)$ and $\mathrm{OS}(\mathrm{HR}=0.66,95 \% \mathrm{CI}=0.45-0.97, P=0.03)$ when chemotherapy was given. With a median follow-up of 10 years, the long-term data reported a $10 \%$ improvement in RFS and a $9 \%$ improvement in OS with the addition of chemotherapy. ${ }^{21} \mathrm{~A}$ high-risk group of patients, defined as stage IB/IC grade $2 / 3$, any stage I grade 3 , or with clear-cell histology might have a greater benefit, with an $18 \%$ improvement in OS reported. These results were consistent with the ACTION Trial, in which patients not fully staged did benefit from adjuvant chemotherapy, but still did not do as well as properly staged patients without adjuvant chemotherapy.

The choice and the duration of chemotherapy for patients with stage I ovarian cancer remains controversial. In the ICON1 study, six cycles of platinum-based chemotherapy were recommended, and $87 \%$ of the patients received singleagent carboplatin. ${ }^{16}$ The retrospective analysis of the ICON3 trial for early-stage ovarian cancer showed a trend toward improved PFS in favor of the combination of carboplatin/ paclitaxel instead of carboplatin alone, but no difference in OS. ${ }^{22}$ The GOG 157 study compared 3 vs 6 cycles of carboplatin/paclitaxel and no difference was shown in RFS or OS between the regimens, although increased toxicity occurred with 6 cycles. $^{23}$
In our study, we have accurate data on chemotherapy available for each individual case, based on an interdisciplinary tumor board decision in accordance with the international guidelines. For patients with FIGO stage IC disease, 82.6\% received platinum-based chemotherapy. The majority of patients who did not receive adjuvant chemotherapy declined it despite our recommendation.

\section{Tumor rupture}

Rosendahl et al suggested that possible explanations for the similar survival rates of substages IA and IC1 were that 1) surgical spill has no significance or 2) patients with stage IC1 disease were treated with adjuvant chemotherapy. ${ }^{9}$ In a large international retrospective study on 1,545 patients, Vergote et al looked at the prognostic factors in stage I invasive epithelial ovarian cancer. In the multivariate analyses, the most powerful prognostic indicator of DSS was the degree of differentiation (poorly vs well differentiated), with a HR of 8.89 (4.96-15.9), followed by rupture of the tumor during surgery with a HR of 1.64 (1.07-2.51), FIGO stage 1973 IB vs IA HR of 1.70 (1.01-2.85), and age (per year HR of 1.02 [1.00-1.03]). ${ }^{24}$ As different adjuvant modalities (chemotherapies vs observation) were permitted in this study, the impact on the outcome remains difficult to appreciate and represents a potential bias. Still rupture of the capsule should be avoided during surgery if possible, ${ }^{25}$ but the data are controversial. It remains unclear whether intraoperative capsule rupture worsens prognosis. .,6,12,26-28 $^{2}$

A recently published meta-analysis reported that intraoperative rupture might not decrease PFS compared to no rupture in patients with early-stage ovarian cancer who undergo complete surgical staging and adjuvant platinumbased chemotherapy. ${ }^{29}$ Furthermore, rupture is often associated with dense adhesions to adjacent tissues, grade 2 or 3 disease, and non-mucinous histology. ${ }^{29}$ These factors are associated with a more aggressive biological tumor behavior.

In our cohort, we recommended a platinum-based chemotherapy to all our patients with FIGO stage IC disease. This may confound the ability to detect any survival differences within the IC subgroups.

\section{Limitations}

Our study has some limitations. The retrospective study design can lead to selection bias. The incompletely staged patients (37.9\%) might represent cases with microscopic metastasis in retroperitoneal lymph nodes that would be upstaged to stage IIIA1 instead of I. The small sample size as well as the small number of events represent a significant limitation to test our hypothesis and explain the lack of statistical power in such a 
retrospective analysis. This represents a true concern for such research question, particularly when reporting data of a rare disease, which becomes even more demanding when split into subgroups. Due to our results and the results and limitations from the literature, we would advise that the question of staging in early-stage epithelial ovarian cancer should be addressed within a large prospective collaborative cohort, for instance within the rare cancer cohort of The European Network for Gynaecological Oncological Trial groups (ENGOT) or even Gynecologic Cancer INtergroup (GCIG). This, however, might not be feasible due to funding issues and it is questionable whether a meta-analysis of published data would shed any further light onto it.

\section{Conclusion}

In the present analysis, we were unable to demonstrate any significant prognostic differences between the subgroups of stage IC in the revised FIGO staging system for ovarian and fallopian tube carcinomas. This may be related to the small study size but also to the fact that $82.6 \%$ of the patients with stage IC disease were given adjuvant chemotherapy. The usefulness of the new FIGO classification in early-ovarian cancer remains still questionable and would need a prospective trial within a trial consortium to be properly answered.

\section{Acknowledgment}

The abstract was presented as poster at the EBCOG meeting 2016 in Torino, Italy, and is available online.

\section{Author contributions}

CMS and VHS contributed to conceptualization; CMS, VHS, $\mathrm{UM}$, and AS involved in methodology; AS contributed to software, validation, and formal analysis; CMS and UM performed investigation; VHS, DF, and $\mathrm{NH}$ are resources; CMS involved in writing - original draft; UM, AS, GM, DF, $\mathrm{NH}$, and VHS contributed to writing - review and editing; CMS, GM, NH, and VHS involved in visualization; VHS contributed to supervision; and CMS contributed to project administration. All authors contributed toward data analysis, drafting and critically revising the paper and agree to be accountable for all aspects of the work.

\section{Disclosure}

The authors report no conflicts of interest in this work.

\section{References}

1. Ferlay J, Parkin DM, Steliarova-Foucher E. Estimates of cancer incidence and mortality in Europe in 2008. Eur J Cancer. 2010;46(4): $765-781$
2. Ahmed FY, Wiltshaw E, A'Hern RP, et al. Natural history and prognosis of untreated stage I epithelial ovarian carcinoma. J Clin Oncol. 1996;14(11):2968-2975.

3. Binder PS, Prat J, Mutch DG. The future role of molecular staging in gynecologic cancer. Int J Gynaecol Obstet. 2015;131(Suppl 2):S127-S131.

4. Young RC, Decker DG, Wharton JT, et al. Staging laparotomy in early ovarian cancer. JAMA. 1983;250(22):3072-3076.

5. Powless CA, Aletti GD, Bakkum-Gamez JN, Cliby WA. Risk factors for lymph node metastasis in apparent early-stage epithelial ovarian cancer: implications for surgical staging. Gynecol Oncol. 2011;122(3):536-540.

6. Prat; JFIGO Committee on Gynecologic Oncology. Staging classification for cancer of the ovary, fallopian tube, and peritoneum. Int $J$ Gynaecol Obstet. 2014;124(1):1-5.

7. Harrell FE, Jr. Regression Modeling Strategies: with Applications to Linear Models, Logistic and Ordinal Regression, and Survival Analysis. 2nd ed. New York: Springer; 2015.

8. Elattar A, Bryant A, Winter-Roach BA, Hatem M, Naik R. Optimal primary surgical treatment for advanced epithelial ovarian cancer [review]. Cochrane Database Syst Rev. 2011;8:CD007565.

9. Rosendahl M, Høgdall CK, Mosgaard BJ. Restaging and Survival Analysis of 4036 Ovarian Cancer Patients According to the 2013 FIGO Classification for Ovarian, Fallopian Tube, and Primary Peritoneal Cancer. Int J Gynecol Cancer. 2016;26(4):680-687.

10. Suh DH, Kim TH, Kim JW, et al. Improvements to the FIGO staging for ovarian cancer: reconsideration of lymphatic spread and intraoperative tumor rupture. J Gynecol Oncol. 2013;24(4):352-358.

11. Paik ES, Lee YY, Lee EJ, et al. Survival analysis of revised 2013 FIGO staging classification of epithelial ovarian cancer and comparison with previous FIGO staging classification. Obstet Gynecol Sci. 2015;58(2):124-134.

12. Zeppernick F, Meinhold-Heerlein I. The new FIGO staging system for ovarian, fallopian tube, and primary peritoneal cancer. Arch Gynecol Obstet. 2014;290(5):839-842.

13. Webber C, Gospodarowicz M, Sobin LH, et al. Improving the TNM classification: findings from a 10-year continuous literature review. Int J Cancer. 2014;135(2):371-378.

14. Maringe C, Walters S, Butler J, et al; ICBP Module 1 Working Group. Stage at diagnosis and ovarian cancer survival: evidence from the International Cancer Benchmarking Partnership. Gynecolo Oncol. 2012;127(1):75-82.

15. Ataseven B, Harter P, Grimm C, et al. The revised 2014 FIGO staging system for epithelial ovarian cancer: Is a subclassification into FIGO stage IVA and IVB justified? Gynecol Oncol. 2016;142(2):243-247.

16. Trimbos JB, Vergote I, Bolis G, et al; EORTC-ACTION collaborators European Organisation for Research and Treatment of Cancer-Adjuvant ChemoTherapy in Ovarian Neoplasm. Impact of adjuvant chemotherapy and surgical staging in early-stage ovarian carcinoma: European Organisation for Research and Treatment of Cancer-Adjuvant ChemoTherapy in Ovarian Neoplasm trial. J Natl Cancer Inst. 2003;95(2):113-125.

17. Timmers PJ, Zwinderman AH, Coens C, Vergote I, Trimbos JB. Understanding the problem of inadequately staging early ovarian cancer. Eur J Cancer. 2010;46(5):880-884.

18. Kleppe M, van der Aa MA, Van Gorp T, Slangen BF, Kruitwagen RF. The impact of lymph node dissection and adjuvant chemotherapy on survival: A nationwide cohort study of patients with clinical early-stage ovarian cancer. Eur J Cancer. 2016;66:83-90.

19. Colombo N, Guthrie D, Chiari S, et al; International Collaborative Ovarian Neoplasm (ICON) collaborators. International Collaborative Ovarian Neoplasm trial 1: a randomized trial of adjuvant chemotherapy in women with early-stage ovarian cancer. J Natl Cancer Inst. 2003;95(2):125-132.

20. Trimbos B, Timmers P, Pecorelli S, et al. Surgical staging and treatment of early ovarian cancer: long-term analysis from a randomized trial. $J$ Natl Cancer Inst. 2010;102(13):982-987. 
21. Collinson F, Qian W, Fossati R, et al. Optimal treatment of early-stage ovarian cancer. Ann Oncol. 2014;25(6):1165-1171.

22. International Collaborative Ovarian Neoplasm Group. Paclitaxel plus carboplatin versus standard chemotherapy with either single-agent carboplatin or cyclophosphamide, doxorubicin, and cisplatin in women with ovarian cancer: the ICON3 randomised trial. Lancet. 2002;360(9332): $505-515$.

23. Bell J, Brady MF, Young RC, et al. Randomized phase III trial of three versus six cycles of adjuvant carboplatin and paclitaxel in early stage epithelial ovarian carcinoma: a Gynecologic Oncology Group study. Gynecol Oncol. 2006;102(3):432-439.

24. Vergote I, de Brabanter J, Fyles A, et al. Prognostic importance of degree of differentiation and cyst rupture in stage I invasive epithelial ovarian carcinoma. Lancet. 2001;357(9251):176-182.
25. Bakkum-Gamez JN, Richardson DL, Seamon LG, et al. Influence of intraoperative capsule rupture on outcomes in stage I epithelial ovarian cancer. Obstet Gynecol. 2009;113(1):11-17.

26. Seidman JD, Yemelyanova AV, Khedmati F, et al. Prognostic factors for stage I ovarian carcinoma. Int J Gynecol Pathol. 2010;29(1):1-7.

27. Chan JK, Tian C, Monk BJ, et al. Prognostic factors for high-risk earlystage epithelial ovarian cancer: a Gynecologic Oncology Group study. Cancer. 2008;112(10):2202-2210.

28. Obermair A, Fuller A, Lopez-Varela E, et al. A new prognostic model for FIGO stage 1 epithelial ovarian cancer. Gynecol Oncol. 2007;104(3): 607-611.

29. Kim HS, Ahn JH, Chung HH, et al. Impact of intraoperative rupture of the ovarian capsule on prognosis in patients with early-stage epithelial ovarian cancer: a meta-analysis. Eur J Surg Oncol. 2013;39(3):279-289.
Cancer Management and Research

\section{Publish your work in this journal}

Cancer Management and Research is an international, peer-reviewed open access journal focusing on cancer research and the optimal use of preventative and integrated treatment interventions to achieve improved outcomes, enhanced survival and quality of life for the cancer patient. The manuscript management system is completely online and includes
Dovepress

a very quick and fair peer-review system, which is all easy to use. Visit http://www.dovepress.com/testimonials.php to read real quotes from published authors. 